\title{
A New Cathodoluminescence System for Nanoscale Optics, Materials Science, and Geology
}

\author{
T. Coenen, ${ }^{1 *}$ S. V. den Hoedt, ${ }^{1}$ and A. Polman ${ }^{2}$ \\ ${ }^{1}$ DELMIC B.V., Thijsseweg 11, 2629 JA Delft, The Netherlands \\ ${ }^{2}$ Center for Nanophotonics, FOM Institute AMOLF, Science Park 104, 1098 XG Amsterdam, The Netherlands \\ *coenen@delmic.com
}

\begin{abstract}
Cathodoluminescence $(C L)$ spectroscopy is a technique that can be used to unravel optical and material properties at the nanoscale. This article describes a novel CL detection system. The system sensitivity, the modularity, and the capability to perform angle-resolved CL imaging enable many new research directions. We introduce the technique and give a description of the setup as well as the different $C L$ emission processes. Subsequently, we show a number of practical examples where the technique is used in the fields of nanophotonics, materials science, and geology.
\end{abstract}

\section{Introduction}

In modern-day technology there is an ongoing effort to miniaturize functional elements in integrated electronics and photonics, light-emitting diodes, solar cells, and sensors for enhanced performance. Associated with this trend is a strong need to perform various forms of microscopy and spectroscopy down to the nanoscale to gain a fundamental understanding about the materials involved, and also for quality control and failure analysis of devices.

Electron microscopy has proven to be an excellent method for obtaining structural information on small-length scales. Traditionally, electron microscopes were designed for imaging transmitted, secondary, and backscattered electrons to provide spatial information down to the atomic scale, and it was generally not possible to characterize the optical properties of a material in this way. Conversely, (light) optical microscopy and spectroscopy provide a means to directly determine optical properties in detail, but these methods are limited in spatial resolution by diffraction.

In CL spectroscopy visible/near-infrared light generated by a fast electron beam is collected and analyzed. The key benefit of this technique is that the high spatial resolution of the electron microscope is combined with the functional optical information that is contained within CL emission. CL spectroscopy has been used for decades in studies of bulk and thin-film materials and now has reemerged as an important tool for nanoscale optical characterization of (nanostructured) materials, largely as the result of recent advances in electron microscopy and light detection.

CL Emission Processes. CL is light in the UV/VIS/ NIR regime emitted from a material under fast free electron bombardment. Interestingly, the discovery and analysis of CL emission in vacuum tubes generated by "cathode rays" played a key role in the discovery of the electron [1]. There are two distinct physical processes that can lead to CL emission, each of which can serve a purpose in particular applications.

Incoherent CL. The first type is categorized as incoherent CL emission where no phase relationship exists between the incoming electron field and the CL emission. This type of CL emission bears a strong resemblance to photoluminescence, as it involves quantum mechanical transitions in a material (see
Figure 1a). Such CL is dominant in phosphors (rare-earth doped materials), direct bandgap semiconductors ( $\mathrm{GaAs}, \mathrm{GaN}, \mathrm{InP}$, etc.), and most dielectrics (geological materials, glasses, ceramics, etc.) [2-4]. Typically, this emission is most efficiently excited by slower electrons, and hence this CL is generated in an interaction volume below the impact position, where the size of the volume is determined by the energy of the primary electrons. As a result one primary electron can generate many photons resulting in a bright CL signal, which forms the basis for efficient electron scintillators and cathode ray tube (CRT) screens. Because of its sensitivity to intrinsic material properties (electronic band gap, defect centers, etc.), this type of $\mathrm{CL}$ is very useful for material fingerprinting, quality assurance, and failure analysis of materials.

Coherent CL. The second type of CL is categorized by coherent interactions between the electron and a material. In this case, the evanescent electromagnetic field of the electron coherently polarizes the material (see Figure 1b). Even though these coherent processes are physically distinct from conventional "luminescence" processes like photoluminescence or incoherent CL, they also fall under the general umbrella term "cathodoluminescence." Electron-induced radiation emission (EIRE) is sometimes used as an alternative term for these coherent processes to avoid confusion with the incoherent form of CL. Examples of coherent excitations include transition radiation (TR), which is generated when an electron traverses an interface between two media; bulk plasmon and surface plasmon polariton (SPP) excitation in plasmonic metals; and Cherenkov radiation, which is generated when the speed of the electron exceeds the phase velocity of light in the material. The advantage of this type of process is that it is very localized $(<20 \mathrm{~nm})$ and fast $(\sim 1 \mathrm{fs})$; as a result it is broadband both
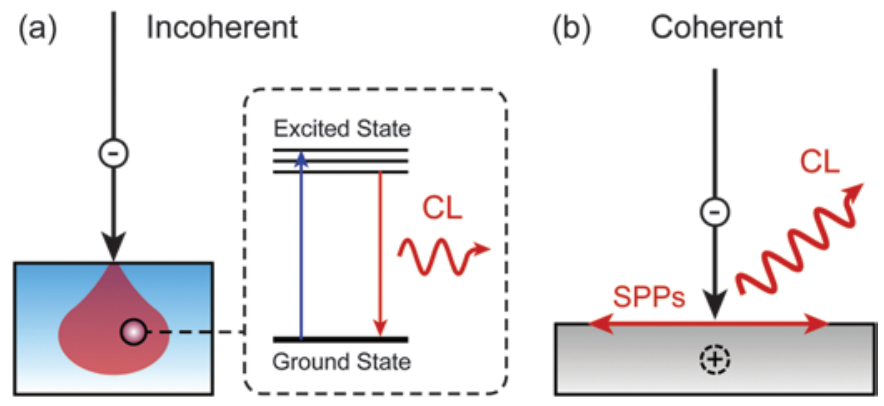

Figure 1: (a) Schematic representation of incoherent CL emission. The electron beam has an interaction volume within the material (indicated in red) in which excitations are generated. Valence electrons are promoted into an excited state after which they can decay with the emission of a photon, as indicated by the schematic Jablonski diagram. (b) Schematic representation of coherent CL emission. The evanescent fields of the electron can locally polarize a material, which leads to direct light emission (TR) and excitation of other supported electromagnetic modes such as SPPs. 

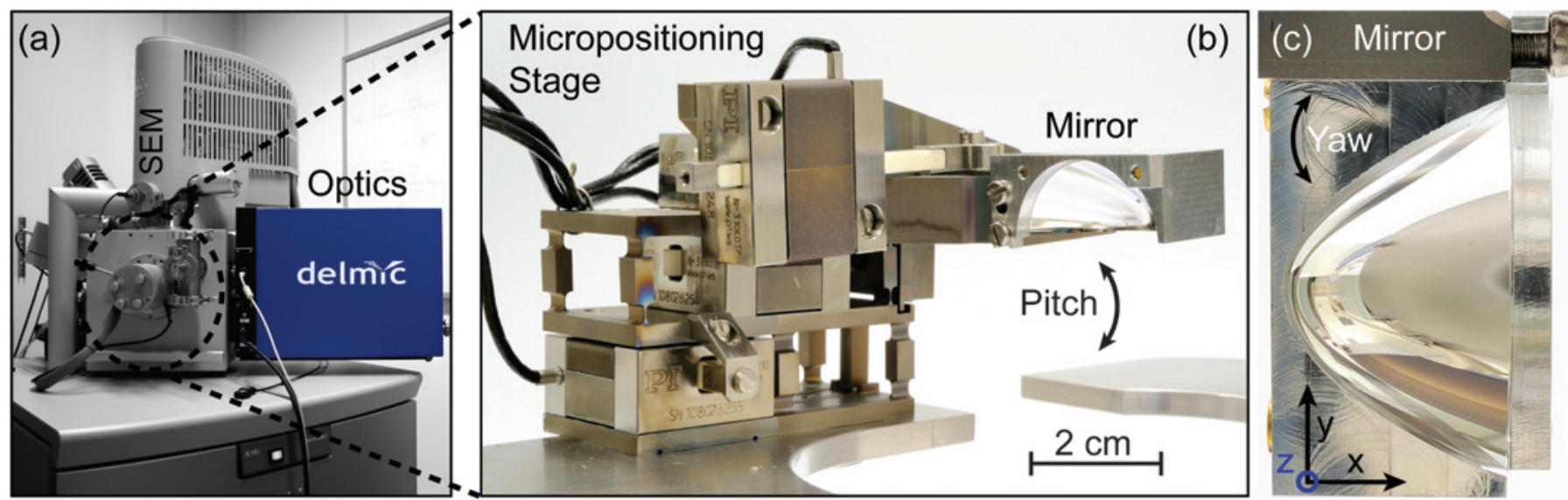

(d)
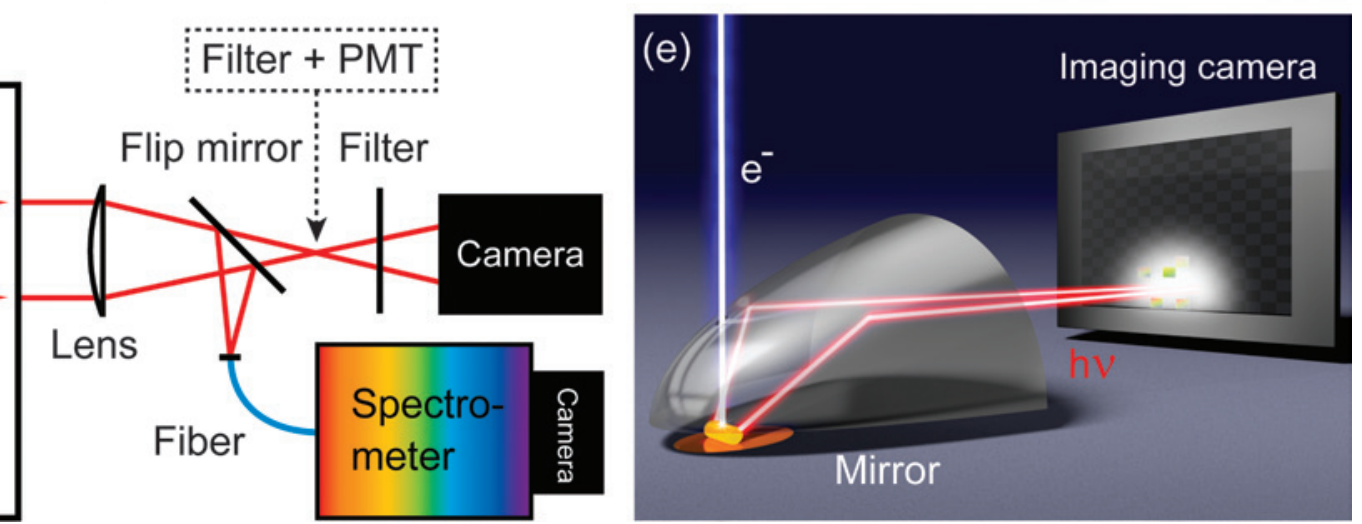

Figure 2: (a) Photograph of FEI Quanta 250 FEG SEM with SPARC CL optics box attached (Chalmers, Sweden). (b) Inside the chamber, a piezoelectric mirror positioning system is mounted, which is used to position a parabolic mirror in four dimensions ( $x, y$, pitch, and yaw). (c) Photograph taken from the bottom of the mirror. (d) Schematic overview of the setup showing the different detection schemes: a spectrometer for 2D CL imaging spectroscopy, a PMT for fast wavelength filtered or panchromatic imaging, and a CCD imaging detector for angle-resolved measurements. (e) Graphical representation of angle-resolved detection of CL on a 2D CCD array (image by Tremani). Photographs in (b, c) by Henk-Jan Boluijt.

in frequency and momentum, allowing the electron to couple to a wide variety of optical modes [4]. Additionally, the excitation probability is related to the local density of optical states (LDOS), a key quantity in optics [5]. Although this type of CL generation is always present, the excitation probabilities are relatively low ( $10^{-4}$ photons per electron), requiring a sensitive detection system and careful sample design. At typical scanning electron microscopy (SEM) currents the sample is bombarded by $\sim 10^{10}$ electrons per second leading to an emission of $10^{6}$ photons per second of coherent CL signal, which is enough for spectroscopy purposes. Hence, such a probe is very useful for studying nanophotonics below the diffraction limit for light.

\section{Materials and Methods}

The SPARC system is a sensitive, modular CL system that is built as a plug-in for a scanning electron microscope (SEM). It was developed at the FOM Institute AMOLF (Amsterdam, The Netherlands), and the technology was then transferred to DELMIC at the end of 2011. Figure 2a shows a photograph of a system installed on a FEI Quanta 250 FEG SEM (Chalmers University of Technology). The system has a motorized light collection system mounted in the SEM chamber (Figure $2 b$ ), in which the micropositioning of a high-quality aluminum paraboloid mirror is performed by piezomotors connected to a titanium leaf-spring system. The mirror can be moved laterally $(x, y)$ and can be rotated in two directions (tilt, yaw) (see Figure 2c).
The alignment in the vertical direction can be done with the SEM stage. Such micropositioning of the paraboloid is necessary to obtain a well-aligned system in which the CL beam coming from the mirror is parallel, ensuring optimal light collection in the optics box outside of the SEM (Figure 2d). Furthermore, the mirror has a large acceptance angle $(1.46 \pi \mathrm{sr})$ to maximize light collection. The combination of these two features gives the system a high sensitivity and allows detection of faint signals such as the coherent forms of CL. The parallel CL beam can be directed to a variety of detectors: Czerny-Turner spectrographs with charge-coupled device (CCD) or complementary metal oxide semiconductor (CMOS) detectors for hyperspectral analysis and photomultiplier tubes for wavelength filtered imaging and/or panchromatic (unfiltered) fast imaging applications. The detectors can be optimized for the wavelength range of interest (ultraviolet, visible, near infrared).

In addition to the spectral imaging in the more conventional CL imaging mode, the SPARC platform can also perform angle-resolved imaging $[6,7]$. In angle-resolved mode the CL beam is projected onto a $2 \mathrm{D}$ CCD array allowing angle-resolved detection of the emission (Figure 2e), that is, the emission intensity as function of azimuthal angle $\varphi$ and zenithal angle $\theta$. By using the proper coordinate transformation, the data collected on the CCD can be converted to units of emitted power per steradian. To obtain spectral selectivity in this 


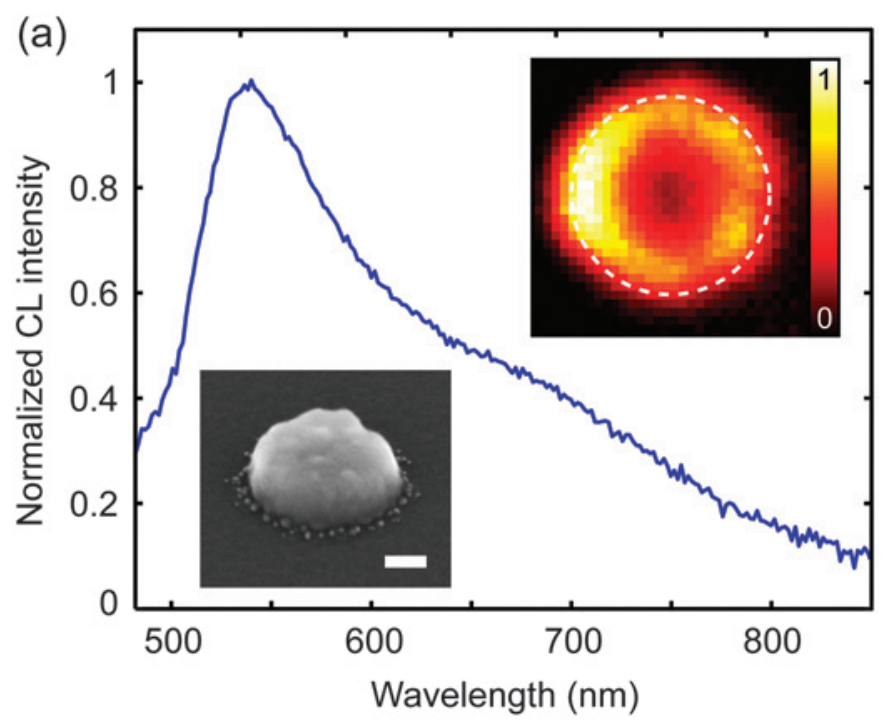

(b)

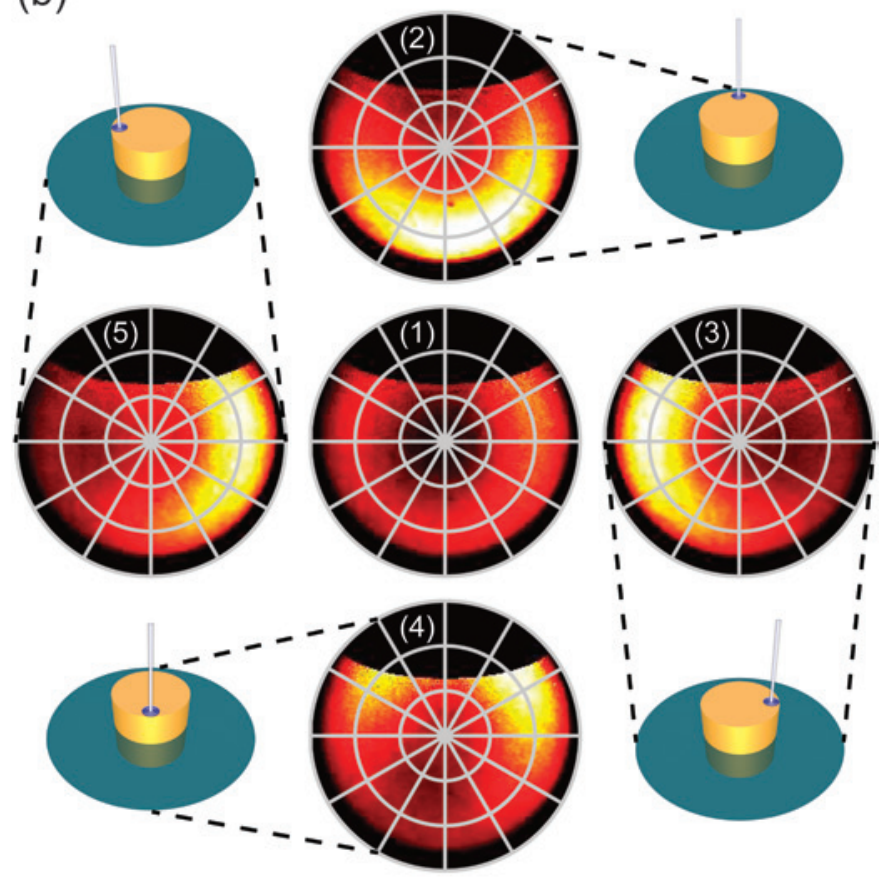

0

1

Figure 3: (a) Normalized CL spectrum from an Au nanodisk on Si with a diameter of $180 \mathrm{~nm}$ and a height of $80 \mathrm{~nm}$. A SEM image is shown as an inset, scale bar $=50 \mathrm{~nm}$. The spectrum is taken by averaging the $\mathrm{CL}$ spectrum over the particle. A $2 \mathrm{D} \mathrm{CL}$ excitation map taken at the peak wavelength $\lambda_{0}=550 \mathrm{~nm}$ is shown as an inset (top right). The dashed line indicates the edge of the structure taken from the SEM image. (b) Experimental normalized angular CL emission patterns at $\lambda_{0}=600 \mathrm{~nm}$ for excitation at the center (1) and near the edge for four orthogonal azimuthal angles: $0^{\circ}(2), 90^{\circ}(3), 180^{\circ}(4)$, and $270^{\circ}(5)$. The edge excitation positions are also indicated by the schematic illustrations in the corners. The patterns have been normalized to the maximum intensity value for all five patterns to show the relative brightness for different excitation positions [13].

angle-resolved measuring mode, band-pass color filters can be used. In the beam path there is space for additional optical components, such as polarizers. This allows reconstruction of the emission polarization for every emission angle and/or polarization-sensitive hyperspectral imaging [8-10].

\section{Applications}

The SPARC system can be used in a variety of fields. Several practical examples from different fields are given below.

Nanophotonics and plasmonics. The field of nanophotonics is concerned with the confinement, generation, detection, guiding, and switching of light at the nanoscale. Plasmonics is a branch of nanophotonics that has received significant interest in the last decade. Plasmons are elementary excitations of free electrons in metals in which light is confined to a metal-dielectric interface. Plasmonic nanostructures have the ability to strongly confine light for spontaneous emission enhancement in antennas, to create optical hot spots for sensing, for enhancement of light absorption in photodetectors and thin-film solar cells, for photothermal therapy, and as transparent conducting nanowire meshes [11].

Figure $3 \mathrm{a}$ (inset) shows an SEM image of a single $\mathrm{Au}$ nanodisk on a Si substrate, made using electron beam lithography and lift-off techniques [12]. The Au nanoparticle is $180 \mathrm{~nm}$ in diameter and has a height of $80 \mathrm{~nm}$. In addition, Figure 3a shows a CL spectrum taken by averaging the CL emission collected from a raster scan over the particle with $7.5 \mathrm{~nm}$ resolution. The spectrum reflects the resonant plasmon modes in the Au nanoparticle. The linewidth of the resonant peak in the spectrum is determined by radiative and Ohmic damping. A spatial map of the CL emission, also shown in Figure $3 \mathrm{a}$, shows that the nanoparticle plasmon is excited most efficiently at the edge, where the electron beam couples efficiently to in-plane dipolar and quadrupolar plasmon modes.

Figure $3 \mathrm{~b}$ shows angular distributions of the radiation emitted by the single Au nanoparticle for central excitation and excitation at four edge positions (as indicated by the illustrations). The CL emission intensity is shown as function of zenithal angle $\theta$ and azimuthal angle $\varphi$. The black region on the top of the patterns corresponds to the opening in the paraboloid mirror (see Figures $2 \mathrm{~b}$ and $2 \mathrm{c}$ ) where no light is collected. While the nanoparticle excited in the center shows a symmetric angular profile reflecting the doughnut-type emission from an upward-pointing dipole, the angular distributions on the edge show a clear asymmetry. For instance, with excitation on the left side of the particle the CL emission is more intense toward the right side of the angular pattern. This asymmetry arises from the fact that off-center electron beam excitation leads to the simultaneous excitation of in-plane and out-of-plane multipole components, which interfere in the far-field [12]. This example demonstrates how angle-resolved CL can be used to study such nanophotonic systems in great detail on length scales that are inaccessible with conventional light microscopy. It should be noted that the technique is not limited to plasmonic systems and can be applied also to dielectric/semiconductor nanophotonic geometries (for example, nanoparticles, photonic crystals) $[4,5]$.

Imaging in 3D: CL tomography. Imaging with $\mathrm{CL}$ usually provides information in 2D. However, it is known from seismic, X-ray, and TEM imaging that tomographic analysis can provide 3D information. In tomography a series of $2 \mathrm{D}$ images is made under different tilt angles after which mathematical algorithms are applied to reconstruct the 3D structure of the data. Such tomographic reconstruction was recently performed with EELS in the context of plasmonic nanostructures [13], but it can also be done with CL 
in which the plasmon excitation occurs in a similar manner.

As proof of principle, CL tomography was performed on a plasmonic crescent consisting of a polystyrene core and a gold shell (see Figures 4a and 4b) [14]. Such crescents have interesting magnetoelectric optical properties and serve as building blocks for metamaterials. Rather than tilting a single crescent, seven crescents with similar dimensions but different orientations were imaged for simplicity. In an alternative method, a virtual tilt series was created from a single measurement using the symmetry of the crescent system. Figure $4 \mathrm{c}$ shows the result of this reconstruction method where the crescent shows a rich $3 \mathrm{D}$ CL structure where the core and base are dominant for blue light. Toward the infrared, the top of the crescents become more dominant because of the strong plasmonic tip mode that exists there.

Materials science. Materials like rare-earth-doped phosphors and III/V semiconductors play an important role in optoelectronic devices, including scintillators, light-emittingdiodes, diode lasers, and solar cells. As mentioned, nanostructuring is employed increasingly for optimization of the optical properties in such materials. CL can be used to study these materials (both in bulk and in nanostructured materials) and to determine their light-emitting properties at the nanoscale. Figure 5 shows CL spectra for a variety of dielectric and semiconductor materials, illustrating the wide scope of materials that can be studied. By combining the spectral sensitivity with the high spatial resolution of the technique, subtle changes in the output spectrum, defect luminescence, angular profile, and emission polarization [10] can be analyzed on a deep-subwavelength scale.

For active CL color impurities, such as rare-earth ions, the detection limit can be as low as $10^{14}$ atoms $/ \mathrm{cm}^{3}$ if the host material is non-absorbing and if there are no competing CL emission lines [3]. This is well below the ppm level and the detection limit of common SEM-based X-ray techniques, making CL spectroscopy a highly valuable technique for visualizing trace element variations.

Geology. The SPARC system is also useful in the field of geology, more specifically in petrology. Petrology is the branch of geoscience that is concerned with the analysis of the origin, distribution, texture, and composition of rocks. Microanalysis of rocks plays a key role in establishing the aforementioned properties. The SEM provides an ideal platform for such studies because it supports different types of imaging modes that can be applied and combined at small length scales $(<1 \mu \mathrm{m})$.

CL has been widely used in geology for decades and is often applied together with energy-dispersive or wavelengthdispersive X-ray spectroscopy (EDS, WDS) [15], as well as secondary electron (SE), backscattering electron (BSE), and electron backscattering diffraction (EBSD) imaging. These imaging modes provide information on elemental composition, topology, density, and crystal structure, respectively. CL microscopy complements these techniques because it is sensitive to elemental composition, to the phases present (crystal structure), and to the presence of defects and trace elements in the material. Such characteristics strongly depend 

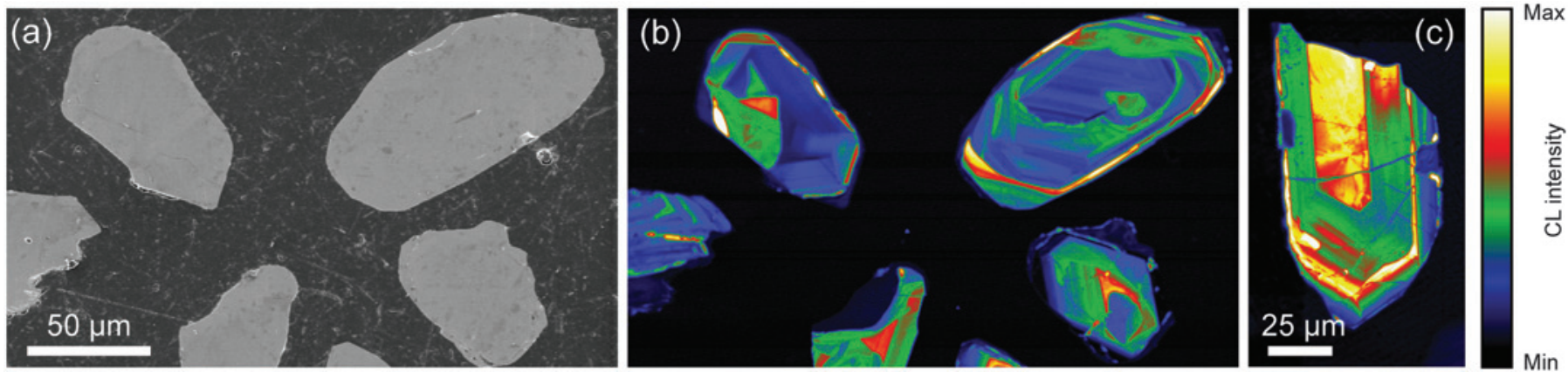

Figure 6: (a) SEM image of some zircon grains. (b) Panchromatic PMT intensity image (false color) of the same area. (c) Close-up panchromatic PMT image of a single zircon grain (measurements by Martijn Haring). These measurements were taken at $10 \mathrm{kV}$ acceleration voltage, $1 \mathrm{nA}$ current, and a dwell time of $100 \mu \mathrm{s}$.

on the conditions under which the rock was formed (chemical composition of the melt, pressure, temperature, presence of water, etc.) and what environments it was exposed to during its lifetime.

Zircons. As an example we a show a measurement on a number of zircon grains in which the CL emission (partly) originates from traces of extrinsic rare-earth-ion color centers [16]. Figures $6 \mathrm{a}$ and $6 \mathrm{~b}$ show an SEM image and a panchromatic CL image, respectively, of the same area. Both figures show zircon grains, but the CL image shows more structure within the grains. As growth conditions change during formation, the zircon is affected. Such changes in conditions lead to zonation in the zircon (zones of zircon that are slightly different in composition), and this can be visualized with CL because the method is sensitive to such small changes [16]. Figure $6 c$ shows another CL image in which there is strong CL contrast within a single grain; here again the zonation is clearly visible. CL is often used as a prescreening tool for high-resolution secondary-ion-mass spectrometry (SIMS) to image the zonation pattern and identify regions of interest (ROIs) for isotope analysis. These regions can then be interrogated with the more expensive and time-consuming SIMS technique. Such an analysis has been used to date zircons that are more than 4.3 billion years old, the oldest native crustal rock of Earth ever to be dated [17].

Sandstone. Sandstone is a sedimentary rock consisting of compacted grains of sand (typically quartz). Sandstone is one of the rock types that can contain oil and gas. Studying the origin and geological history of such rocks with CL spectroscopy can therefore play an important role in
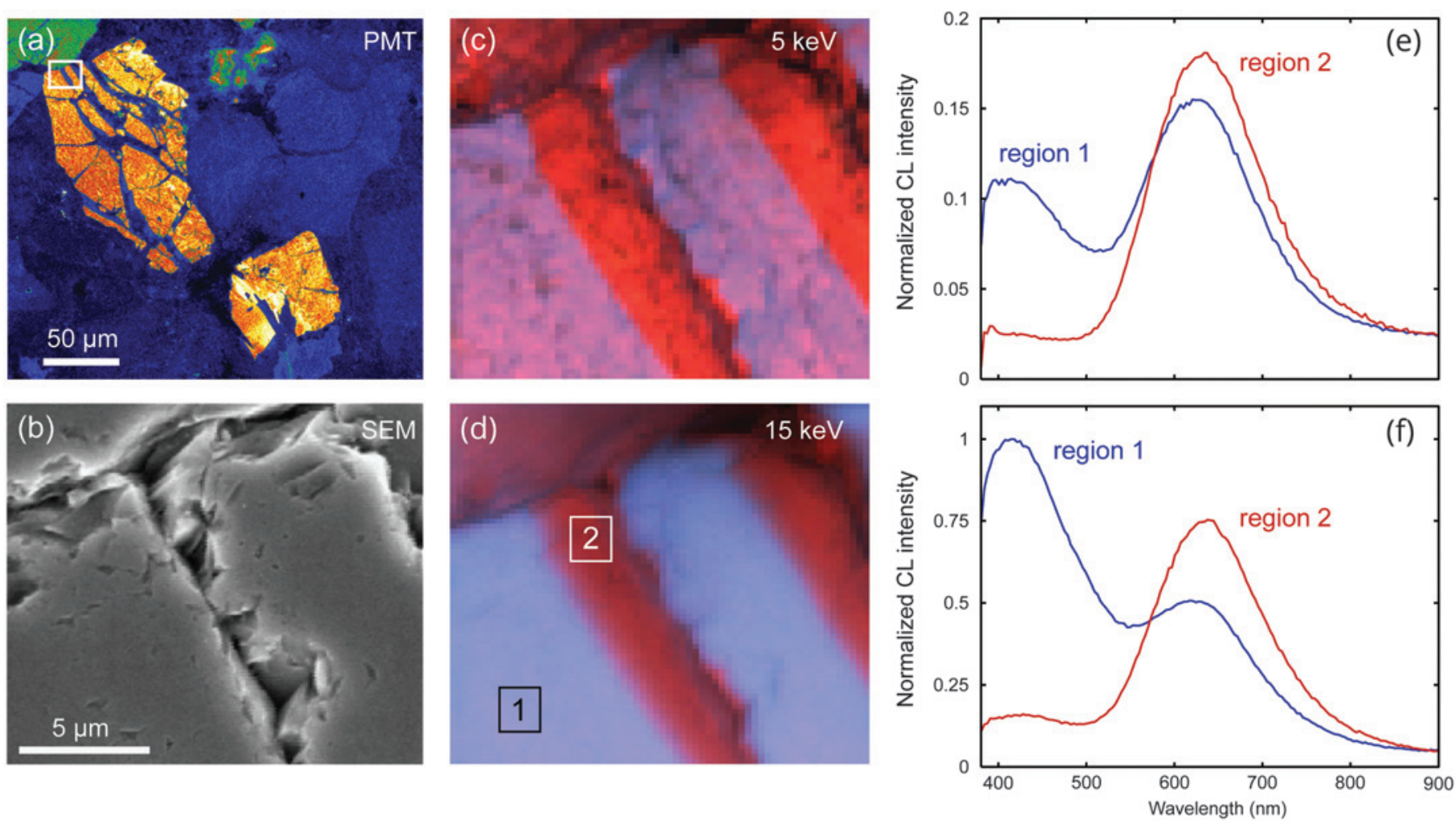

Figure 7: Sandstone analysis. (a) PMT image (5kV) that is used to identify a ROI (indicated by the white box). (b) SEM image of the ROI from (a). (b) and (c) show false-color RGB images derived from the CL datacube acquired from the area with $80 \mathrm{~ms}$ dwell time at $5 \mathrm{kV}$ (c) and $15 \mathrm{kV}$ (d), respectively. The color coding is blue $=390-476 \mathrm{~nm}$, green $=477-563 \mathrm{~nm}$, and red $=564-650 \mathrm{~nm}$. (e) and (f) show spectra taken from the regions indicated in (d) for $5 \mathrm{kV}$ (e) and $15 \mathrm{kV}(\mathrm{f})$. 
evaluating the economic potential of deposits [18]. In particular, CL spectroscopy is useful in distinguishing plutonic quartz (that is, the high-quality detrital grains) from lower-grade quartz cements by their distinct CL signatures.

A typical CL workflow for sandstone is shown in Figure 7. Figure $7 \mathrm{a}$ shows a panchromatic photomultiplier (PMT) image that exhibits an interesting CL contrast on a fractured grain. Subsequently, a ROI is selected from this image to perform a more in-depth spectral analysis. To visualize the contrast in the hyperspectral CL data we have made false-color RGB images where the spectral region from 390 to $650 \mathrm{~nm}$ is separated in 3 sections that are binned such that the total intensity in these spectral regions defines an RGB code. Figures $7 \mathrm{c}$ and $7 \mathrm{~d}$ show such images for 5 and $15 \mathrm{kV}$ acceleration voltage. In both cases there is strong contrast revealing regions with strong "blue" and "red" emission
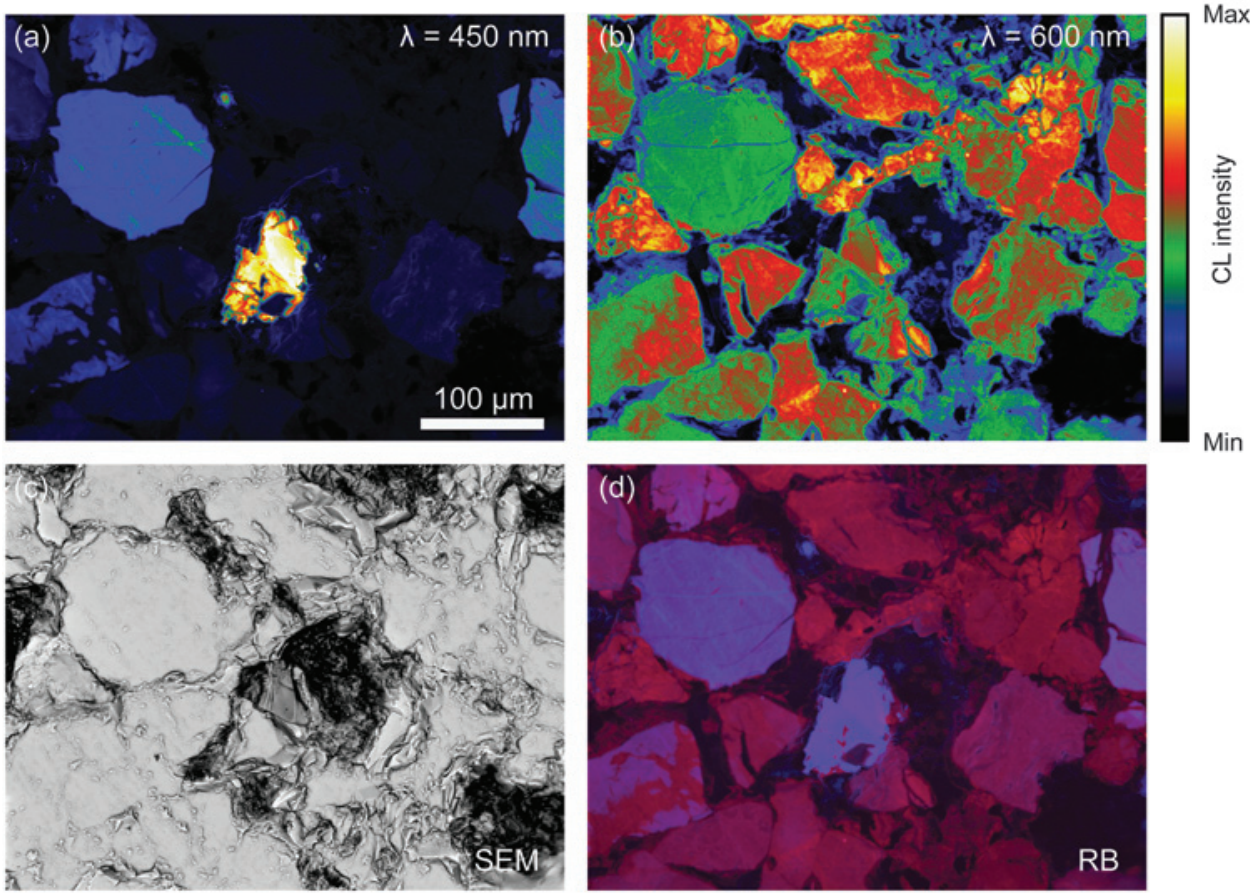

Figure 8: Quartz analysis. Single-wavelength PMT measurements taken with a (a) $450 \mathrm{~nm}$ and a (b) $600 \mathrm{~nm}$ band pass filter with a $40 \mathrm{~nm}$ bandwidth. (c) SEM image of a sandstone sample corresponding to the region inspected with the PMT. (d) Red-blue color image in which the PMT images in (a) and (b) are placed in the blue and red color channel, respectively. For these measurements the conditions were $40 \mathrm{nA}$ beam current, $15 \mathrm{kV}$ acceleration voltage, and a $40 \mu$ s dwell time. that are completely invisible in the

SE image (Figure 7b). We also note that the colors are not entirely the same for the two acceleration voltages and that the image for $15 \mathrm{kV}$ is more blurry and uniform, reflecting a larger excitation volume. In (e) and (f) we show spectra for the two distinctly colored regions as indicated in (d) at 5 and $15 \mathrm{kV}$, respectively. Consistent with the color coding in (c, d) we find that the blue peak at $\lambda=410 \mathrm{~nm}$ is dominant at region 1 whereas the red peak at $\lambda=640 \mathrm{~nm}$ dominates in region 2 . These peaks are associated with intrinsic defects in the $\mathrm{SiO}_{2}$ crystal matrix [19]. Their different relative magnitude indicates that these are two different quartz types: the blue emitting quartz corresponds to the larger cracked detrital crystal grain whereas the red emitting quartz corresponds to cement that has infilled the cracks [18].

Knowledge about the distinct spectral properties of different quartz types can be used to perform fast segmentation of larger areas by performing color-filtered PMT imaging. The images in Figures $8 \mathrm{a}$ and $8 \mathrm{~b}$ correspond to CL images from the same area but taken with different color filters that are roughly matched to the two peak positions of Figure $7 \mathrm{f}$. Such color-filtered images can be combined into a multicolor image as shown in Figure 8d (in this case the images in (a) and (b) are mapped onto the blue and red color channels, respectively). Such false coloring can be used for any combination of color filters and helps to visualize CL features.

These segmentation studies provide a means to quantitatively map the quartz composition of a rock sample. By combining such results with in-depth analysis of the observed textures such as fractures and grain contacts, the geological history as well as the porosity or permeability of the rock can be established. While such studies are of fundamental interest, they are also used by the petroleum industry for assessment of reservoir rocks.

\section{Discussion}

The motorized parabolic mirror system in the SPARC allows precise alignment of the optical system, thus greatly boosting the collection effiency and enabling measurements on low-light-emitting samples such as the metallic nanostructures mentioned above. Additionally, in cases where the incoherent form of CL is studied, it allows one to employ lower acceleration voltages, in which the CL yield per incoming electron is reduced, but the spatial resolution improves because of the smaller interaction volume [3] (see Figures $7 \mathrm{c}$ and 7d). Because the alignment procedure in the SPARC can be done deterministically using the 2D imaging camera, it is highly reproducible. Hence, light output can be quantitatively compared between samples, allowing for growth-process optimization and optical quality assurance. By studying a material with a known CL emission like aluminum and measuring the probe current, the absolute system response can be recorded, enabling comparison of the CL emission in different materials in absolute terms (that is, the absolute number of photons emitted per electron) [20].

The ability to perform angle-resolved measurements, including a full study of CL emission polarization, adds a whole new dimension to CL research enabling a large number of CL applications. The angular profiles and polarization sensititivity can be used to study directionality of nanostructures $[6,8,12]$, dispersion in (a)periodic photonic crystals [5], and chiral (handed) structures [10], and to rigorously separate coherent and incoherent CL contributions [10, 20]. 
Furthermore, the optics and detectors of the SPARC system can in principle be adapted for other spectral regimes such as the deep UV, which is relevant for dielectrics, high-bandgap semiconductors, or the mid-infrared which corresponds to the spectral regime where phonons and vibrational modes in materials can be probed. Additionally, the development of CL tomography opens up new avenues for obtaining spectroscopic information on the nanoscale in 3D. Besides tomography, other types of 3D CL imaging can also be employed in which focused-ion-beam (FIB) milling is used to make cross sections of stratified media or is even used to perform in-situ slice-andview experiments [21]. This method could be particularly useful in the context of thick extended structures for which it is hard to use projection-based tomographic approaches.

Finally, we envision that the SPARC platform could be extended to study the time and temperature dependence of CL. These additions would provide great experimental tools for performing more in-depth spectroscopic analysis on a large variety of materials, studying photon emission correlation statistics in single quantum emitters [22], and probing the local density of optical states in nanophotonic environments through emitter lifetimes.

\section{Conclusion}

We have shown that the SPARC system provides a versatile platform for nanoscale CL studies in a variety of research fields including nanophotonics, materials science, and geology. As the nanotechnology research field and industrial market continues to grow, the demand for advanced nanoscale characterization and microscopy will increase further, leading to a bright future full of challenges and questions for which the SPARC system can provide innovative solutions.

\section{Acknowledgements}

This article reviews work done in collaboration with Ernst Jan Vesseur, Benjamin Brenny, Ashwin Atre, Aitzol García-Etxarri, Jennifer Dionne, Felipe Bernal Arango, and Femius Koenderink. We would like to acknowledge Prof. Jens Jahren (University of Oslo) for providing the zircon sample and Eric Goergen (FEI Company) for providing the sandstone sample. Technical support, design, and fabrication from Hans Zeijlemaker, Ilya Cerjak, Wim Brouwer, and Jan van der Linden for the AMOLF prototype are gratefully acknowledged. The DELMIC team is acknowledged for the design of the commercial SPARC system. The AMOLF part of this work is part of the research program of the Foundation for Fundamental Research on Matter (FOM), which is financially supported by the Netherlands Organization for Scientific Research. It is also supported by the European Research Council and by NanoNextNL, a research program funded by the Dutch Ministry of Economic Affairs. Toon Coenen is an employee of DELMIC B.V. Albert Polman and Sander den Hoedt are co-owners and founders of DELMIC B.V.

\section{References}

[1] W Crookes, Philos Trans 170 (1879) 641-62.

[2] M Pagel et al., Cathodoluminescence in Geosciences, Springer, New York, 2000.

[3] BG Yacobi and DB Holt, Cathodoluminescence Microscopy of Inorganic Solids, Plenum Press, New York, 1990.

[4] FJ García de Abajo, Rev Mod Phys 82 (2010) 209275.

[5] R Sapienza et al., Nat Mater 11 (2012) 781-87.

[6] T Coenen et al., Nano Lett 11 (2011) 3779-84.

[7] T Coenen et al., Appl Phys Lett 99 (2011) 143103.

[8] T Coenen and A Polman, Opt Express 20 (2012) 18679-91.

[9] CI Osorio et al., ACS Photonics 3 (2016) 147-54.

[10] BJM Brenny et al., Appl Phys Lett 107 (2015) 201110.

[11] JA Schuller et al., Nat Mater 9 (2010) 193-204.

[12] T Coenen et al., Nat Commun 5 (2014) 3250.

[13] O Nicoletti et al., Nature 502 (2013) 80-84.

[14] A Atre et al., Nat Nanotech 10 (2015) 429-36.

[15] CM MacRae et al., Microsc Res Tech 67 (2005) 271-77.

[16] JM Hanchar and PWO Hoskin, Reviews in Mineralogy and Geochemistry 53 (2003) 469-500.

[17] SA Wilde et al., Nature 409 (2001) 175-78.

[18] S Boggs, Jr. and D Krinsley, Application of CL Imaging to the Study of Sedimentary Rocks, Cambridge University Press, New York, 2006.

[19] MA Stevens Kalceff and MR Phillips, Phys Rev B 52 (1995) 3122-34.

[20] BJM Brenny et al., J Appl Phys 115 (2014) 244307.

[21] DAM de Winter et al., J Microsc 243 (2011) 315-26.

[22] LHG Tizei and M Kociak, Phys Rev Lett 110 (2013) 153604.

\section{Precision, Speed, Stability NANO-POSITIONING FOR MICROSCOPY}

\section{PI}

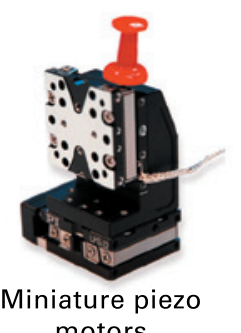

motors

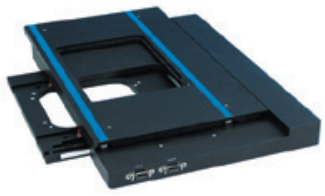

High-stability $X Y$ piezo motor stage, $100 \mathrm{~mm}$

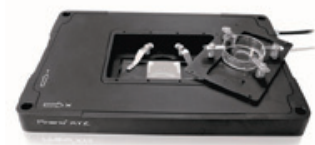

$X Y Z$ flexure stage $<1 \mathrm{~nm}$ resolution

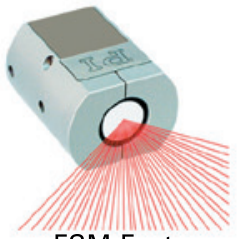

FSM Fast Beam Steering

PI (Physik Instrumente) LP · Auburn, MA · info@pi-usa.us · 508-832-3456 PRECISION MOTION CONTROL \& POSITIONING SOLUTIONS 


\section{SPI Supplies UV Prep ${ }^{\mathrm{mI}}$ for SEM}

\section{UNPrep" FOR SEM}
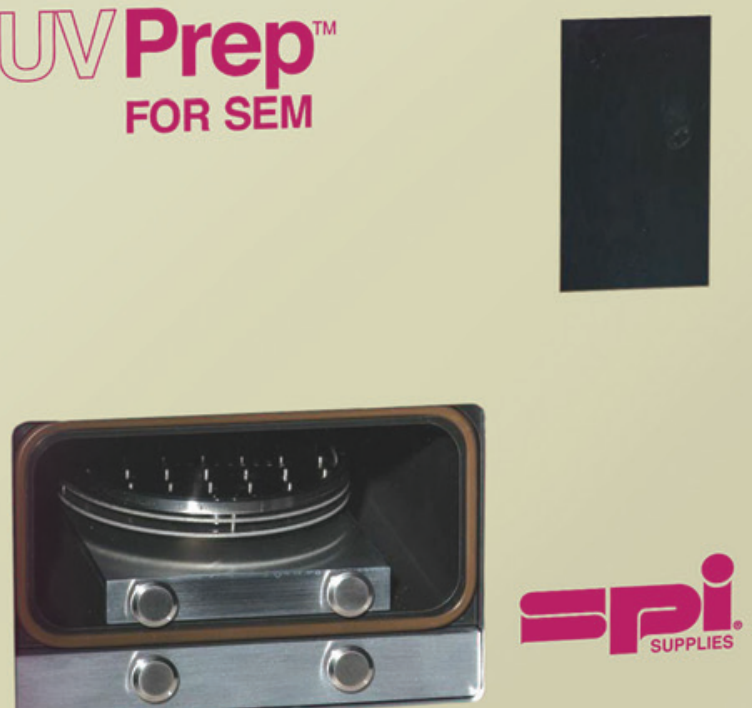

The UV Prep removes surface hydrocarbon contamination to reveal enhanced surface detail not otherwise visible in the SEM just a click away...2spi.com/uvp

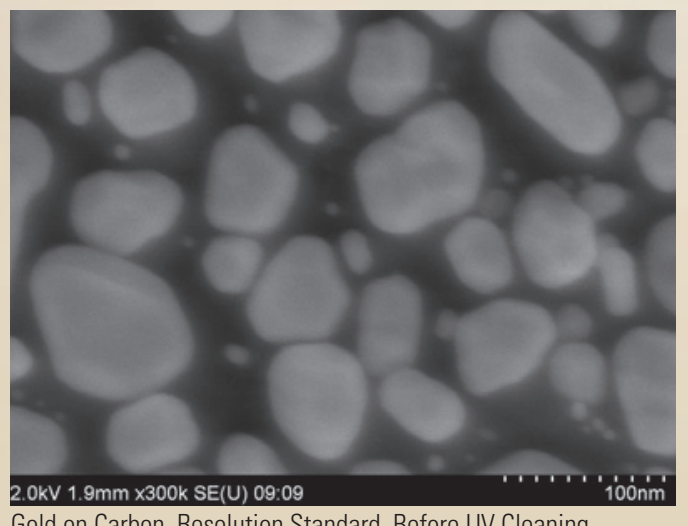

Gold on Carbon, Resolution Standard. Before UV Cleaning.

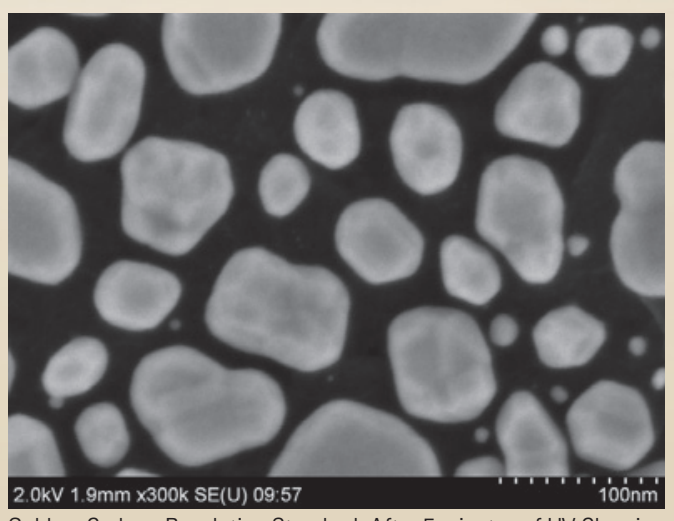

Gold on Carbon, Resolution Standard. After 5 minutes of UV Cleaning.

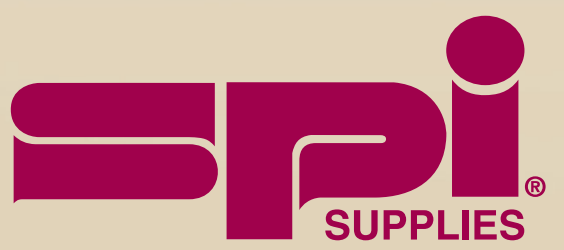

SPI Supplies Division of STRUCTURE PROBE, Inc. 